\title{
Assessing the feasibility of mobile phones for follow- up of acutely unwell children presenting to village clinics in rural northern Malawi
}

\section{Victoria Hardy ${ }^{1}$, Jenny $\mathrm{Hsieh}^{2}$, Baxter Chirambo ${ }^{3}$, Tsung-Shu Joseph $\mathrm{Wu}^{2}$, John O'Donoghue ${ }^{4}$, Adamson S. Muula ${ }^{5}$, Matthew Thompson ${ }^{1}$}

\author{
1. Department of Family Medicine, University of Washington, Seattle, Washington, USA \\ 2. Luke International (LIN), Malawi Office, Mzuzu, Malawi \\ 3. Faculty of Health Sciences, Mzuzu University, Mzuzu, Malawi \\ 4. Global eHealth Unit, Department of Primary Care and Public Health, Imperial College London, London, United Kingdom \\ 5. Department of Public Health, College of Medicine, University of Malawi, Blantyre, Malawi
}

Correspondence: Miss Victoria Hardy (vhardy4@uw.edu)

\section{Background}

\section{Abstract}

Patient follow-up is a routine component of clinical practice and valuable for evaluating the effectiveness of interventions, but because of the broad dispersion of health facilities and lack of standardised medical reporting in Malawi, collecting patient outcome data can be challenging. Increasing accessibility and affordability of mobile technology in resource-poor settings may facilitate patient follow-up in the community. The objective of this study was to evaluate the potential utility of mobile phones for collecting follow-up clinical data from parents or caregivers of acutely unwell under-5 children, for intervention evaluation purposes.

Methods

Parents' or caregivers' mobile phone numbers were obtained by health surveillance assistants (HSAs) during study enrollment. Guardians who provided a telephone number were contacted by the study team to establish re-consultations or hospitalisations of their child(ren) within 14 days of recruitment. Health records at village clinics and higher-level health facilities were hand-searched to identify or confirm presentations and abstract clinical data.

Results

87 out of $149(58.4 \%)$ guardians provided a mobile telephone number, of whom the study team could contact $44(29.5 \%)$. Seven guardians stated they took their child for further treatment: three of these returned to village clinics and four presented to secondary care facilities; attendance could only be confirmed from health records for one child.

\section{Conclusions}

With continued expansion of cellular network coverage and mobile ownership in Malawi, mobile phones may facilitate collection of patient outcomes for intervention evaluation purposes. Future consideration should also be given to integrating mobile technologies into HSA clinical practice.

\section{Introduction}

Community case management (CCM) supports frontline health workers, called health surveillance assistants (HSAs) in Malawi, to treat children under five years of age presenting with uncomplicated infections and to refer those with more serious illness to higher-level facilities. ${ }^{1}$ Patient follow-up is part of routine clinical practice and is important for monitoring illness progression and continuity of care. ${ }^{2}$ However, extant literature suggests children in some regions of sub-Saharan Africa, who would benefit from higherlevel care, are not being referred or are failing to present, increasing their risk of severe morbidity and mortality. ${ }^{3,4}$

Knowledge of HSA referral practices, and subsequent patient outcomes post-consultation, permits evaluation of the effectiveness of interventions to improve care, ${ }^{2}$ but it is unclear how measurable these outcomes are in Malawi. Collecting follow-up data is dependent on the ability to accurately identify patients from medical records, which is limited by lack of routine use of a national patient identification system, geographically dispersed health facilities, and a poor medical records infrastructure, which is largely paper-based.

As part of a feasibility study exploring the use of a mobile phone-based application of CCM, called the Supporting LIFE electronic Community Case Management App (SL eCCM App), by HSAs in village clinics in Malawi, we sought to determine whether mobile phones could be used to follow-up acutely unwell children.

Outcomes included the number of parents who provided a mobile phone number, and the proportion of these who were able to be contacted by the research team and provided information on subsequent health service contacts that their child experienced within 14 days of recruitment.

\section{Methods}

A convenience sample of 12 HSAs from Mzimba North recruited parents or guardians of children who presented consecutively to 12 village clinics (one HSA per clinic) during normal opening hours, between July and September 2015. HSAs assessed children (following written consent), first using the SL eCCM App, then again according to standard practice, which directed treatment. Guardians were asked to provide a mobile phone number (if they had one), which was recorded on the consent form. Follow-up telephone calls were conducted by a researcher within 21 days of recruitment to determine subsequent follow-up visits at village clinics, and attendances at health facilities within 14days of recruitment. Three attempts were made to contact guardians, after which they were documented as lost to follow-up. Information given by guardians was used at health facilities for review of medical records.

Patient records at facilities specified by guardians, and facilities located in the catchment area of participating village clinics, were systematically reviewed to ascertain HSA referral decisions at the initial visit, dates of further attendances of children whose guardians did not have a phone, and to confirm guardian-reported information for those successfully contacted by telephone. Ethical approval was granted by the College of Medicine Research and Ethics Committee in Malawi and by the University of Washington, USA. 


\section{Results}

Twelve HSAs recruited a total of 160 children, of whom 11 were later excluded because they fell outside the age range (between 2 months and 5 years of age) for which CCM is validated. Of the 149 included children, 87 (58.4\%) guardians provided a mobile phone number, of whom the study team were able to contact $44(29.5 \%)$. Despite no HSA referrals at the initial clinic visit, seven guardians with mobile phones who were contacted stated that they took their child for further treatment: three reported returning to village clinics and four to secondary-care facilities. Records could only be found for one child at the relevant facility. For children of guardians without a mobile phone, there were no confirmed additional patient visits.

\section{Discussion}

To our knowledge, this is the first study conducted in Malawi that has demonstrated the potential utility of mobile phones for clinical follow-up of acutely unwell children from village clinics. Over half of parents provided a mobile number, of whom half were successfully contacted, providing an overall telephone follow-up rate of 44/149 (29.5\%). Reported access to a mobile phone was considerably less than the $89.3 \%$ cited for patients in urban areas in low- and middle-income countries (LMICs). ${ }^{5}$ This difference may be attributable to lower affluence, poorer accessibility to mobile phones and network coverage in rural areas. ${ }^{5}$ In some cases, guardianreported information could not be substantiated from health records, making it difficult to determine the most valid source of follow-up data. We did not categorically record absence of a mobile phone, which may have resulted in under-reporting of the number of participants with mobile phones.

\section{Conclusions}

Although our sample size was small and our findings may not be generalisable, the expansion of network coverage and mobile phone ownership in Malawi may facilitate the use of mobile phones as a simple method to ascertain outcomes in children recruited to clinical studies in rural areas, and consideration should be given to the role of mobile phone solutions for patient follow-up as part of HSA clinical practice in Malawi.

\section{Acknowledgements}

This study received funding from the European Union's Seventh Framework Programme for research, technological development and demonstration under grant agreement no 305292.

\section{References}

1. WHO/UNICEF. Joint Statement: Integrated Community Case Management (iCCM). 2012. http://www.unicef.org/health/files/iCCM_ Joint_Statement_2012.pdf

2. Wootton R, Liu J, Bonnardot L. Quality Assurance of Teleconsultations in a Store-and-Forward Telemedicine Network - Obtaining Patient Followup Data and User Feedback. Frontiers in Public Health. 2014;2:247.

3. Bossyns P, Abache R, Abdoulaye MS, Miyé H, Depoorter A-M, Van Lerberghe W. Monitoring the referral system through benchmarking in rural Niger: an evaluation of the functional relation between health centres and the district hospital. BMC health services research. 2006;6(1):1-7.

4. Peterson S, Nsungwa-Sabiiti J, Were W, Nsabagasani X, Magumba G, Nambooze J, et al. Coping with paediatric referral-Ugandan parents' experience. The Lancet. 2014;363(9425):1955-6.

5. House DR, Cheptinga P, Rusyniak DE. Availability of mobile phones for discharge follow-up of pediatric. Emergency Department patients in western Kenya. Peer J. 2015;3:e790. 